\title{
JUST IN TIME DYNAMIC \& COST-EFFECTIVE MAINTENANCE (JIT DMAINT) FOR MORE RELIABLE PRODUCTION: A CASE STUDY
}

\author{
Basim Al-Najjar * \\ School for Engineering, Linnaeus University, Linnaeus, Sweden
}

Using CM, we have past data for describing damage initiation \& development and current data for describing the current condition of a component/machine. Data describing possible behaviour of the condition in the close future are usually lack in the available commercial systems although it is important for planning production/maintenance actions, reducing failures/accidents, \& consequently reducing losses \& production cost. In general, accurate maintenance decisions prolong life length of components/machines and maintain production continuity which adds competitive advantages. When CM parameter exceeds a significant (warning) level, it demands a clear understanding of what happened to avoid failures. Also, reliable answers concerning; what is the probability of failure of a component, its residual life and when is the most profitable time of conducting maintenance are necessary.

In this paper, a new innovative eMDSS consists of three strategies for cost-effective maintenance \& production processes is introduced and discussed. The major focus is given on one strategy (Accurate Maintenance Decisions). This strategy offers opportunity to achieve Just on Time Dynamic \& Cost-effective Maintenance by selecting the most profitable time for maintenance is developed, tested in case companies and discussed. It offers unique solutions to increase maintenance profitability by enhancing maintenance decision accuracy via;

1. Predicting the value of CM parameter, e.g. vibration, level, PreVib.

2. Estimating the probability of failure at the close future and component residual life, ProLife.

3. Using this new information, it will easily estimate the most profitable time for maintenance action.

Accurate Maintenance Decisions provides data about future situation in addition to the current \& past data, which is necessary for production and maintenance successful planning. This toolset; predicts the vibration level (PreVib), assesses the probability of failure of equipment and its residual life (ProLife), and consequently it becomes possible to determine the best time of maintenance action. It has been tested in several Swedish and European companies within the branches of car manufacturing, production machine manufacturer and process industry. This study was partly funded by EU-IPDYNAMITE (Dynamic Decisions in Maintenance) 2005-2009 and partly by E-maintenance Sweden AB. The major conclusions are; applying this toolset it is possible to reduce failures appreciably, prolongs the life length of components/equipment, and perform profitable maintenance.

Keywords: Just in Time Maintenance, dynamic and Cost-effective maintenance, prediction of vibration level, probability

\section{INTRODUCTION}

Maintenance plays a key role in reducing production cost through enhancing availability and extending the life length of producing assets, [08] Wu et al. (2007). Using condition monitoring (CM) technologies, production security and operating safety will increase because the prob- ability of detecting and treating problems will increase [09] Wang (2002), [04] Al-Najjar (2001) and Wu et al (2007). Furthermore it will result in much lower operational costs, [07] Xiaodong et al. (2005).

When using CBM system, there are many analytical tools for interpreting the signals and assessing the condition of the component, [04] 
Prediction of the vibration level in the close future utilising previous and current measurements, current and future operating conditions, the predicted value may support and enhance the accuracy of maintenance decisions. But even if it provides reliable information about the condition of a component, we do not certainly know when the component will break, [02] Al-Najjar (1997). Replacing components at unreliable assessment of its condition leads to two types of losses; either losing a big part of its residual life or at failures.

Accurate maintenance decisions influence the economic losses arise due failures in producing companies especially where stoppage time is expensive, [06] Holmberg et al. (2010), chapters 12 and 13. Components, equipment \& machines are usually exposed to changes in their conditions/status during operating time. But when significant changes occur in the condition of a component/equipment it demands a clear understanding of what happened to avoid failures. Knowing the root causes behind changes eases selecting, planning and conducting maintenance actions, [03] Al-Najjar (2000). Also, reliable answers concerning; what is the probability of failure? How long it may survive? And when is the most profitable time of conducting maintenance? Provide reliable underlying information demanded for profitable maintenance decisions, i.e. decision of when to stop the machine for maintenance.

The hard competition in the market forces companies to press down production cost in order to expand the possibility of offering their customers lower price and gain additional competitive advantages. Production process tools, machinery and working environment change dynamically in time while we use static model for maintenance. Thus, it is necessary for production and maintenance managers/engineers that maintenance should be dynamic to be suited for the changes in the production process cost-effectively to enable high decision accuracy for achieving Just in Time Maintenance. One way to do that is to enhance the accuracy of maintenance decisions so that we can select the most profitable time for conducting maintenance action. In this paper, a new maintenance toolset (Accurate Maintenance) for achieving just on time dynamic and cost-effective maintenance by selecting the most profitable time for maintenance is developed, applied and dis- cussed. It offers unique solutions to increase maintenance profit by enhancing maintenance decision accuracy.

\section{PRODUCTION AND MAINTENANCE INTERACTIONS}

From Figure. 1, it is obvious how improvements vibration-based maintenance (VBM) interacts production, quality, production cost, environment, etc. If we follow the arrow started from each of the improvements identified in the Figure 1., a long series of actions can be noticed.

For example, improvement in the accuracy of setting the alarm levels, diagnosis and prognosis and assessment of the time to maintenance action generate different advantages enhancing the outcome of maintenance, such as, less failures, shorter stoppages, longer production time, more production, better working environment, etc. as it shown clearly from Figure 1.

In this paper, the objectives of competitive production with respect to maintenance are, together with companies, specified in following:

- Better utilised company resources.

- Continuous reduction of unplanned stoppages and failures.

- Continuous shortening of downtime.

- Applying profitable maintenance.

- Continuous reduction of production economic losses.

- Continuously enhanced production profitability.

The system that will enable achieving the above mentioned objectives should be specified by the following features:

- Detecting problems at an early stage for following development and preventing failures

- Conducting reliable vibration signal analysis and vibration spectrum analysis.

- Predicting the condition of significant components/equipment in the close future which is necessary for accurate and reliable production and maintenance planning.

- Reliable judgment/evaluation of the component/machine condition and its capability of production according to the production plan.

- Continuous assessment of the economic importance of maintenance and its investments and their contribution in company profit.

- Identifying, selecting and following up the most profitable investments in maintenance. 


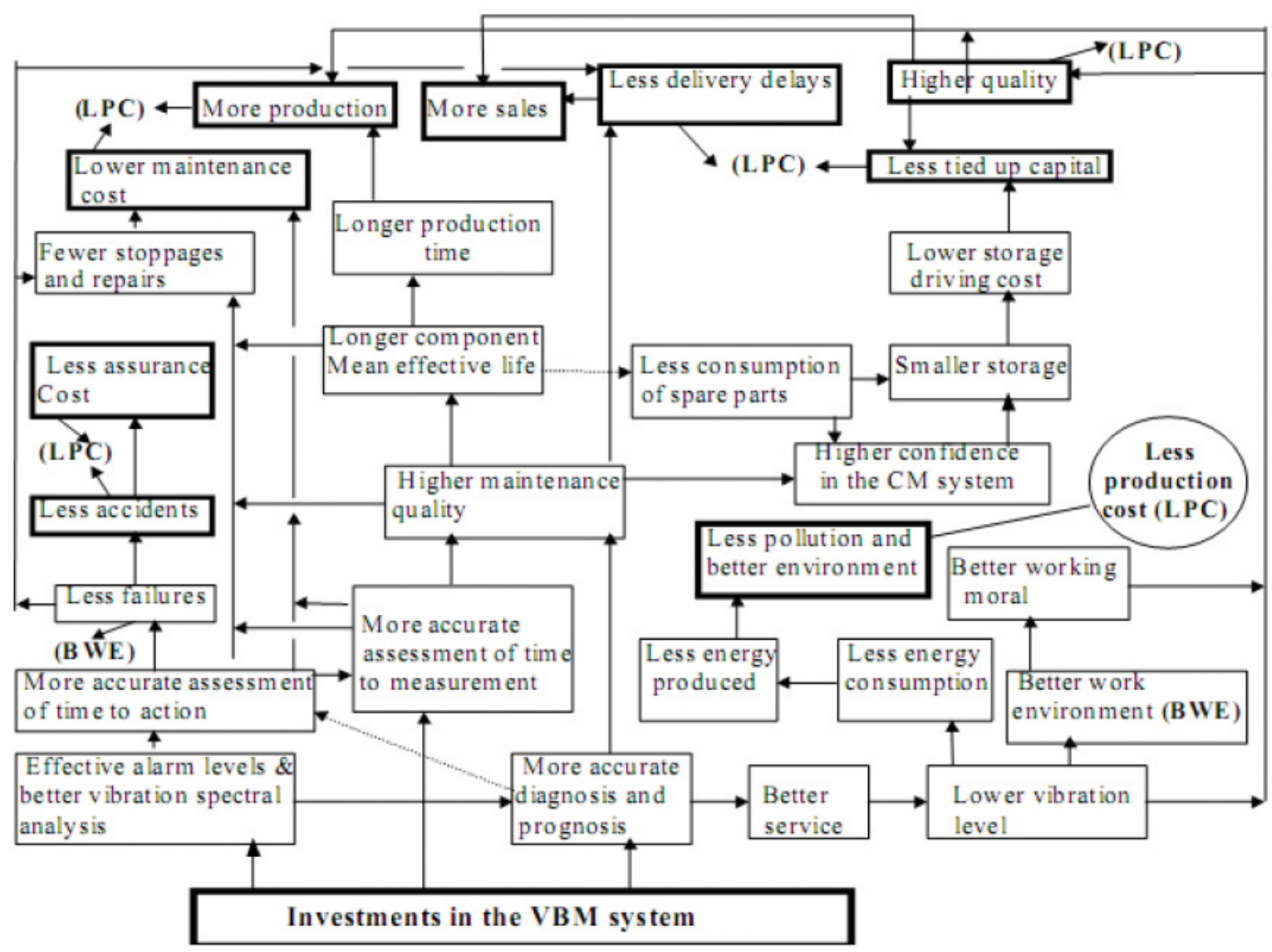

Figure 1: VBM impact on production outcome.

\section{ACCURATE MAINTENANCE DECISIONS}

Using available CM technologies, in many cases, it is possible to tell what has happened in a component/equipment, where and why, but in order to enhance maintenance decision accuracy more information is still demanded, such as reliable answers of:

1. What is the residual life of the component in question, i.e. the probable time that has been left until failure)?

2. What is the probability of failure of the component during the coming period?

3. What is the predicted value of the CM parameter, e.g. vibration level, in the next future, e.g. next measuring opportunity or next planned stoppage?

4. What are, if there any, alternative solutions for the problem in question?

5. What is the most profitable time for conducting maintenance?

6. According to which factors/parameters maintenance is profitable?
Dynamic e-maintenance realised in eMDSS introduces three different strategies for continuous improvement of the cost-effectiveness of maintenance and production processes, see also Figures $1 \& 2$ :

1. Accurate maintenance decisions.

2. Selection of the most cost-effective solution.

3. Follow up maintenance performance and investments.

In this paper, we focus on the strategy for more accurate maintenance decisions and selection the most profitable time for maintenance actions. Assessing the residual time of a component is always necessary for the production manager to check the possibility of fulfilling delivery schedule. While, the probability of failure of the same component is important to assess the chance of proceeding running the production without increasing the risk of failure. Prediction of, e.g. the vibration level during the next future, is for describing the ability of machine to run in the near future without exposing the machine security, operator safety, product quality, working 
environment and may be even production cost to dramatic or catastrophic changes, [01] Al-Najjar (2009). Furthermore, following static rules for judging dynamically changed situation makes the process of decision making rigid and unable to suit dynamically changed situations. This is why these additional information parameters increase the possibility of applying dynamic and cost-effective e-maintenance. In many cases, it is necessary to realise the technical and economic importance of the changes in the technical parameters, e.g. stoppage hours, number of failures, average stoppage time per each short stoppage, lower quality rate, productivity, etc. on the economic scale.

\begin{tabular}{|l|l|l|}
\hline \multicolumn{2}{|c|}{ eMDSS } \\
\hline $\begin{array}{l}\text { Module 1 } \\
\text { Accurate Decisions }\end{array}$ & $\begin{array}{l}\text { Module 2 } \\
\text { Selection of the } \\
\text { most profitable }\end{array}$ & $\begin{array}{l}\text { Module 3 } \\
\text { Following up }\end{array}$ \\
\hline \begin{tabular}{l|l|} 
PreVib \\
Prediction of vibration \\
level (deterioration level)
\end{tabular} & $\begin{array}{l}\text { AltSim } \\
\text { Simulation \& } \\
\text { selection of the } \\
\text { most profitable } \\
\text { maintenance } \\
\text { action }\end{array}$ & $\begin{array}{l}\text { Mapping, following } \\
\text { up and assessing } \\
\text { maintenance } \\
\text { contribution in } \\
\text { company profit }\end{array}$ \\
\hline $\begin{array}{l}\text { ProLife } \\
\text { Assessment of } \\
\text { probability of failure } \\
\begin{array}{l}\text { AN } \\
\text { residual time }\end{array}\end{array}$ & \\
\hline
\end{tabular}

Figure 2: eMaintenance Decision Support System modules and functions.

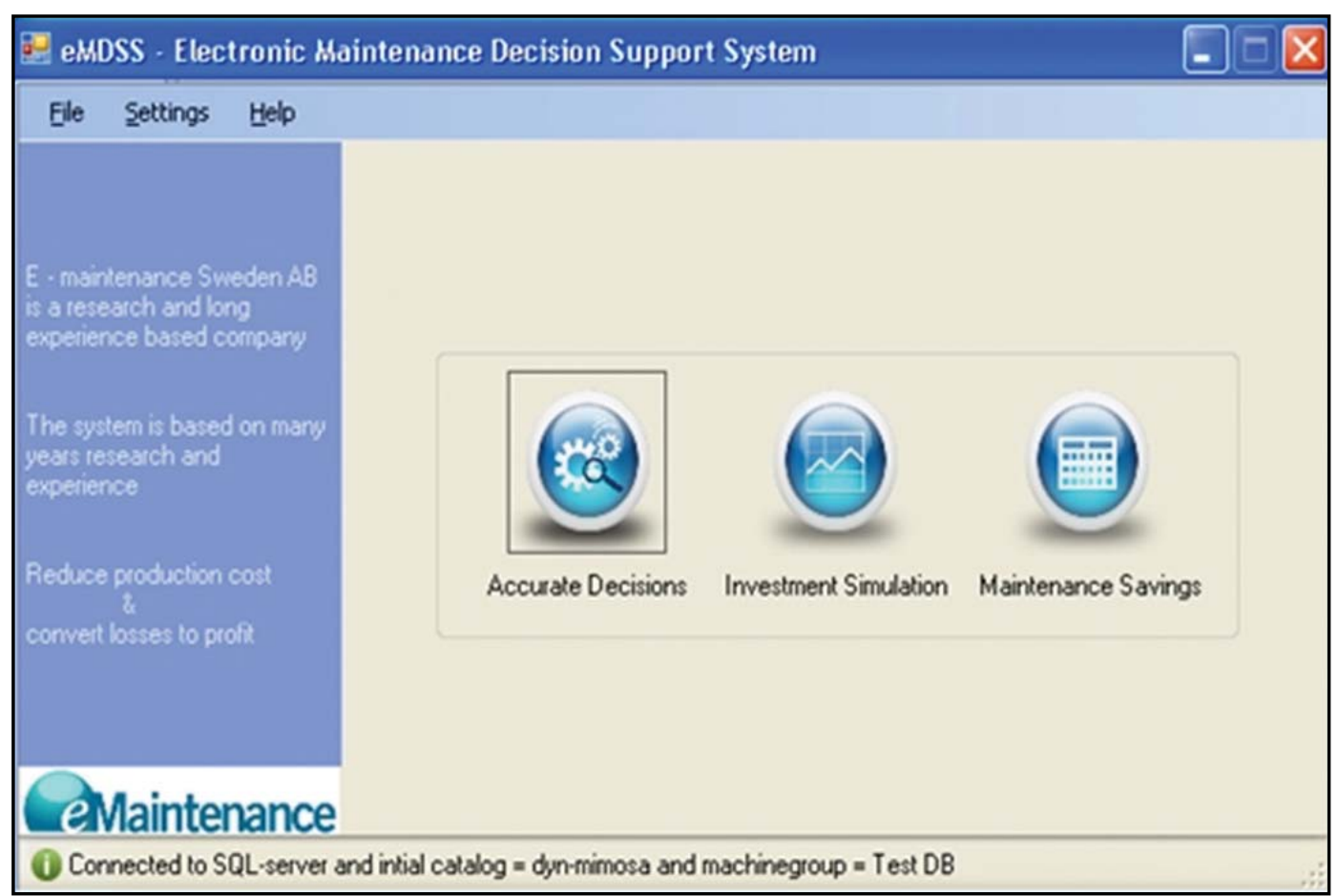

Figure 3: Software programme; eMaintenance Decisions Support System (eMDSS).

Development of PreVib software module is for daily use and to ease the achievement of reliable and faster prediction of the vibration level. Also, ProLife was developed for assessing the probability of failure of a component and its residual lifetime. The user-interface of PreVib is divided into two halves, Fig.3: left half representing input data needed for prediction and right half representing the results in form of a graph. Input data show both database and non-database data in the grey and white boxes, respectively. When a prediction is to be made, the segment 
(machine), asset (component, e.g. a rolling element bearing) and the location (direction of the vibration measurements) should be specified. Also, we can specify how many measurements in "Limit measurement" that we consider if we focus only on part of the measurements. Otherwise, it considers all the measurements for prediction.

Then, the measurements can be downloaded by pressing "Load" which uploads the data from a database (can be MIMOSA). The "Near future load/present load" can specified otherwise it is taken equal to 1. "Mean vibration level (xo)" and "Prediction time period" are specified automatically based on the loaded data. The desired future "Prediction time period" can be specified in, e.g. seconds, hours, days, weeks, months, etc. at need. When "Predict" is clicked, the value of the predict vibration level and the associated date and plot it on the diagram on the right hand side of the user interface will be determined. By analogy, the same thing can be repeated any time the user need to predict the vibration level, for example after the next measurement of the vibration level. The right half of the user-interface presents a graph will contain two plots, the blue representing the actual vibration level values and the yellow showing the predicted vibration level values. Y-axis is the vibration level in, e.g. $\mathrm{mm} / \mathrm{sec}$ and $\mathrm{x}$-axis is the calendar time for measurements and predictions. The graph contains also three horizontal dashed lines representing the three different levels of life of a mechanical component i.e. mean vibration level (xo), potential failure level (xp) and replacement level (xth). These levels are determined and set based on data from identical components and is automatically retrieved when component data is uploaded. Some of the initial measurements belong to the first phase will not be shown in the graph, even if they have been considered when estimating model's constants. The same three levels are also shown in the trend diagram that include all the measurements even those did accepted by PreVib.

The first prediction time should be done after confirming damage initiation, i.e. the vibration level exceeds or close to $\mathrm{xp}$, because as long it fluctuates around xo it means there is no damage is initiated and thereby there is no need for predicting future level. To be able to predict future vibration level one need at least four measurements. This is why we need some of the measurements that have been considered part of the first phase of the component life.

Observe that sometimes, the first two predictions are special cases due to the use of the measurements below the potential failure level to assess model's parameters. The reason for that is; we want to predict future state as soon as we have confirmed the initiation of damage, instead of waiting for at least three measurements exceeded (xp). Waiting could also cause uncertainty in the maintenance decisions. Also, because deterioration process is a stochastic process, vibration level can be changed randomly in time. Therefore, some of the level values may acquire higher level than it should, i.e. exceeds $x p$, which it may be irrelevant to the severity of the deterioration. For each prediction, all previous measurements are used for estimating model's parameters and thus predicted vibration level itself.

ProLife software module is supplementary to PreVib module for enhancing the accuracy in maintenance decisions, which can be used jointly and independently. As for PreVib, ProLife user-interface is also divided into two halves, Fig.4. The left half represents input data required for assessment and in the right half the result is shown in form of a plot (graph). When assessing the probability of failure and residual lifetime of a component, it demands to specify the "Segment" (machine) and "Asset" (component, e.g. a rolling element bearing in this case) and "Assessment time point", i.e. the time at which the assessment should be done followed by clicking assess-button. Subsequently the program uploads the table of lifetime data from the database and presents the graph on the right half of the user-interface.

The $y$-axis in the graph shows the proportion of the average exhausted lifetime and the $x$-axis shows the probability of failure for the analysed component. "Probability of Failure" of the component in question and its "Residual lifetime" are then assessed and displayed in the left hand side of the user-interface. The values can be shown by using the cross-air pointer in the graph. If a new assessment is performed after the current assessment time point, then consequently the cross-air pointer will move forward on the curve. Each point on the graph represents each and one of the component lifetimes in relation to each other. 
The result of the analysis of these two modules, PreVib and ProLife, can be saved for future use as a knowledge- and database. Also, the system can automatically save PreVib information of a particular component when it is replaced as soon as a button in ProLife is pushed. When this is pushed a new windows for ProLife will also be opened for the new component. In this way a complete and accurate register will be accomplished showing the life cycles of the components, installation and replacement times, component previous behaviour (trend), interactions between real measurements and predicted values of vibration levels, etc. which eases accomplishment of continuous improvement of the knowledgebase and database.

\section{CASE STUDY}

The case study is conducted in a Swedish paper mill. The company has three paper machines and the mill is characterised by producing different types of paper which demands different production settings and production speed. The company suffers of some problems related to maintenance, which are;

1. Lack of relevant data needed for more accurate \& dynamic maintenance decisions.

2. Unnecessary stoppages that reduce production time and leads to economic losses.

3. Lack of technique, tools \& knowledge concerning how to assess, follow up and control maintenance economic impact on company profit.

The case study is started by data gathering from the company for mapping the situation. The general comments that can expressed after data analysis are:

- The mill uses vibration for monitoring the condition of rotating components/ equipment and machines through about 3500 measuring points.

- The CM system lacks of warning and replacement levels which in turn makes efficient evaluation and judgment of the condition of any component almost not possible.

- The behaviour of the vibration measurements in time, i.e. trend of vibration level, which usually used for detecting and following up damage initiation and development also lacks.
- The number of measurements per year, which is one of very important elements in any measuring policy is confused and not determined for each measuring point. This makes detection of deviations in the condition of a component at an early stage very difficult to achieve.

- The mill receives analysis report prepared by a service supplier. The report does not assist maintenance personnel in deciding where to conduct maintenance action, when and why. Also, it lacks of the relevant diagnosis highlighting what happened and what may happen.

- The technical and economic data required for mapping, following up, analysis and evaluation of maintenance and production performances are spread in at least three systems if we do not count personal memories. These data are also necessary for obtaining a holistic view of a producing machine. Furthermore, they are necessary to visualize changes in the life cycle of identical and similar components, and past, current and future situations which crucial for cost-effective and dynamic decisions.

- It was not easy to gather all the data required, because it demanded long time. One of the major reasons behind that is; these data were not needed before although most of them are available in different databases. The same thing can be said concerning the information associated with replacement or repair of different components in the machines.

- The losses in the production time are divided in many categories based on the reasons behind these losses, for example mechanical, electrical, instrument, unplanned stoppages, down- and up running of the machine at each stoppage. These categories stand for the biggest part of the losses in the production time.

- Cleaning and preparing of the machine also stands for a big part of the losses in the production time.

- Lack of the data required for highlighting the interactions between economic losses in production, stoppages, downtime due to failures, failure causes, maintenance and production performances. 


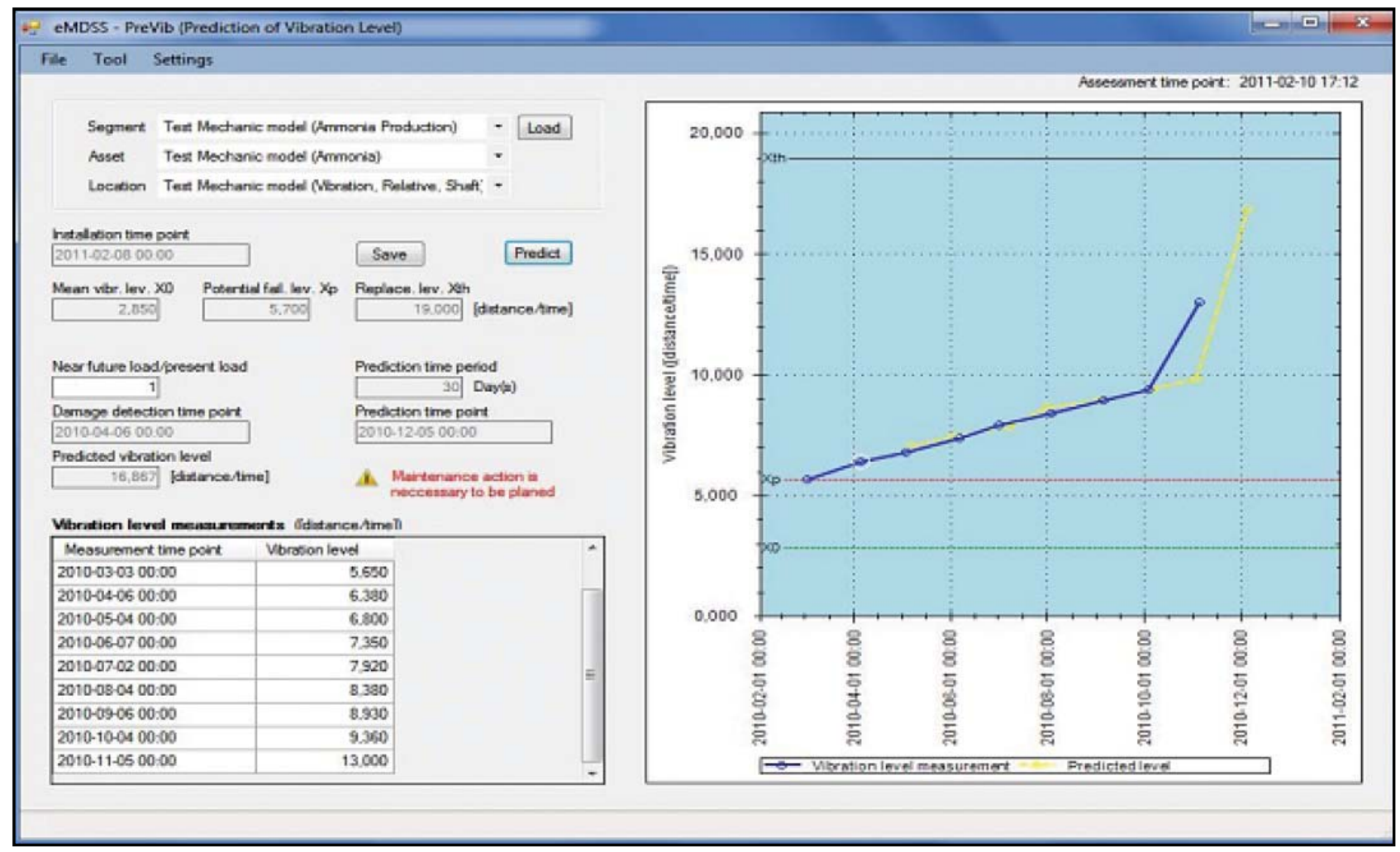

Figure 4: PreVib application

- Lack of the data and techniques required for following up and assessing the economic losses in production and maintenance impact on production, quality and company profit in the past, current and close future. The latter is very important in the strategic planning of an enterprise.

The system characteristics that the company is needed are, in collaboration with company's personnel, specified below, i.e. it should enable the company to:

- follow up the changes in the condition of significant components, i.e. trend.

- provide the relevant data that describe current condition of these components.

- predict the vibration level in the close future, e.g. next planned stoppage or measuring opportunity, i.e. future condition.

- assess the probability of failure of component and its residual life in the close future,i. e. future condition.

- assess the life cycle of components for comparison and effective following up.

- gather and clearly present the information concerning current and future condition of a component.

- Also, we together with the company's per- sonnel identified the need for additional knowledge and experience that are usually required for:

- Assessment of availability, and Overall Equipment Effectiveness (OEE).

- Development of a measuring and analysis policy for determining when, why, where and how, etc. to conduct measurements at different measuring point.

- Effective vibration analysis \& diagnosis as well as effective following up problem development.

- Effective and accurate setting and continuous improvement of alarm levels.

- Identification of the causes behind failures and unplanned stoppages.

\section{RESULTS OF APPLYING PreVib \& ProLife/eMDSS}

eMDSS has been applied using the data gathered from the company. In order to separate eMDSS implementation from the surroundings and neutralise its effect, eMDSS is applied as a standalone system. The major results that have been achieved by this application are:

1. Applying PreVib \& ProLife shown it provides; 
a. Accurate prediction of the deterioration level of a component measured by, e.g. vibration level, in the next future, such as next planned stoppage, see Fig.3.

b. Reliable assessment of the probability of failure of a component and its residual life time, see Fig. 4.

2. eMDSS provides relevant data concerning; live cycle, risk of failure, residual life time, description of the condition in the past, current and in the close future situation, which makes planning and conducting maintenance actions can be done cost-effectively, see Fig.s 3 \& 4 .

3. Decision accuracy is improved continuously based on more measurements.

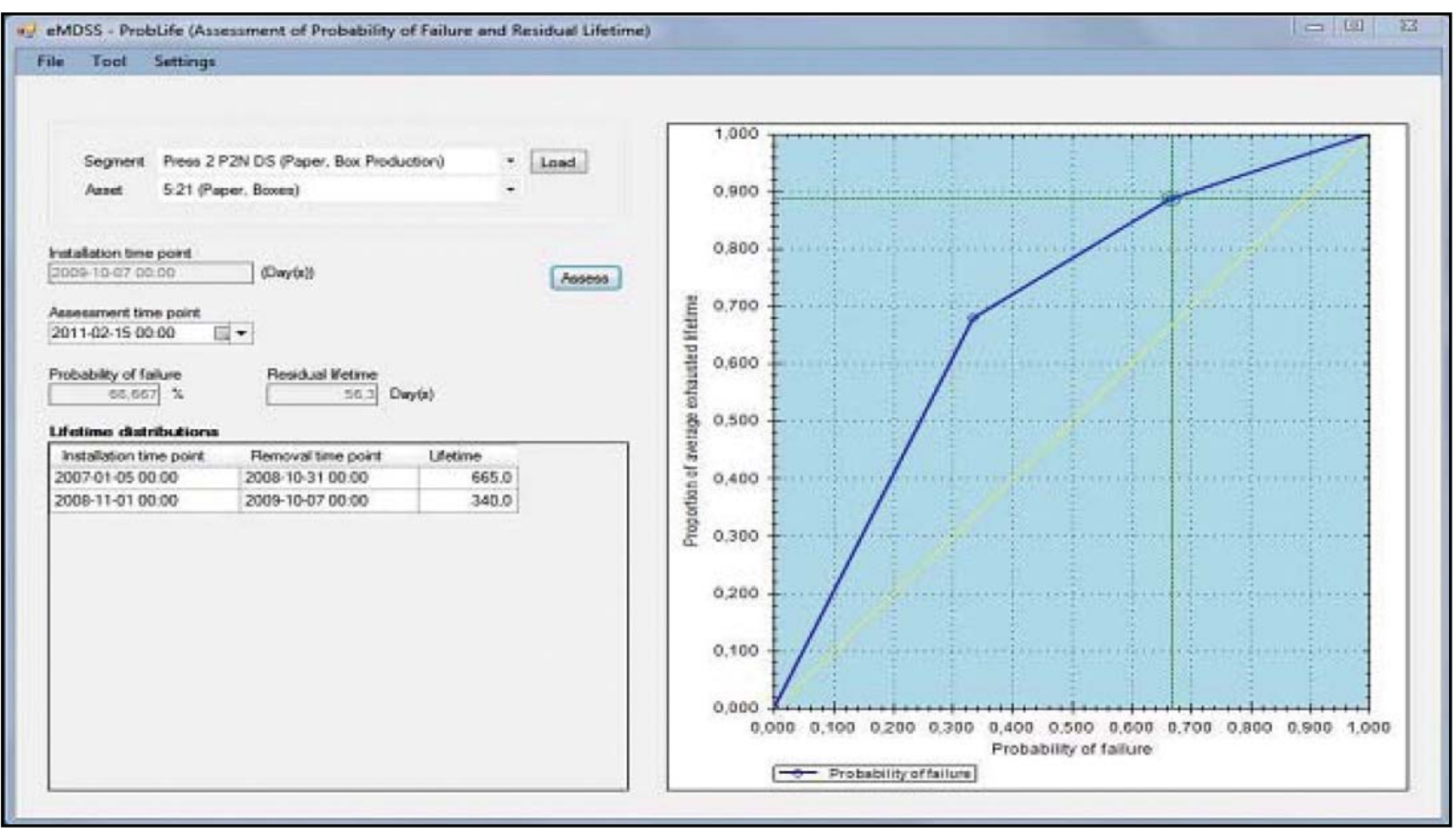

Figure 5: ProLife application

\section{CONCLUSIONS}

Using eMDSS (module; Accurate Decisions), it is possible to act at an early stage in both tactical and strategic levels for fulfilling company's strategic goals in continuous improvement of availability and consequently profitability. Applying this module makes it possible to handle real-time data, analysis and decisions and give necessary information about maintenance and other working areas to the decision maker. It provides better data coverage and quality which are essential for improving knowledge and experience in maintenance and thereby aid in increasing the profit of the company. Predicting the CM parameter level at the next planned measuring time or next planned stoppage reduces the risk of failures. This means that the probability of failure of a component, such as a rolling element bearing can be kept very low until damage is initiated and under development, given that damage can be detected at an early stage through using an efficient CM system. This would reduce the number and duration of planned and unplanned stoppage that has a direct impact on the company productivity. Using eMDSS different tools/software modules there is no duplication of data gathering. Also, eMDSS's modules are flexible and can used in different companies, for different machines, components and products. It is also possible to show how maintenance affects the profit of a company in a way that is hard to do with the available tools and techniques even if the data required are available. Development of relevant and traceable KPIs for technical and economic maintenance control has made this possible. Furthermore, eMDSS does not demand more data than that already available in the company and it does not replace current maintenance systems, but it is a complementary part with unique functions that current maintenance systems do not have. 


\section{ACKNOWLEDGEMENT}

This study was partly funded by EU-IP DYNAMITE (Dynamic Decisions in Maintenance) 2005-2009 and partly by E-maintenance Sweden $A B$.

\section{REFERENCES}

1) Al-Najjar B (2009) A computerised model for assessing the return on investment in maintenance: Following up maintenance contribution in company profit. WCEAM 2009, page 137-145, Greece, Athens.

2) Al-Najjar, B. (1997). Condition-based maintenance: Selection and improvement of a costeffective vibration-based policy in rolling element bearings. Doctoral thesis, ISSN 0280-722X, ISRN LUTMDN/TMIO-1006SE, ISBN 91-628-2545-X, Lund University, Inst. of Industrial Engineering, Sweden.

3) Al-Najjar, B. (2000) Accuracy, effectiveness and improvement of Vibration-based Maintenance in Paper Mills; Case Studies. Journal of Sound and Vibration, 229(2), p. 389-410.

4) Al-Najjar, B. (2001) Prediction of the vibration level when monitoring rolling element bearings in paper mill machines. International Journal of COMADEM 4 (2), p. 19-27
5) Al-Najjar, B. (2003) Total Time on Test, TTTplots for condition monitoring of rolling element bearings in paper mills. International Journal of COMADEM 6 (2), p. 27-32.

6) Holmberg, K., Jantunen, E., Adgar, A., Mascolo, J., Arnaiz, A. and Mekid, S. (2010) E-Maintenance. Springer-Verlag London Limited 2010.

7) Xiaodong, Z. and Xu, R. and Chiman, K. and Liang, S.Y. and Qiulin, X. and Haynes, L. (2005). An integrated approach to bearing fault diagnostics and prognostics, American Control Conference, 2005. Proceedings of the 2005, p. 2750-2755

8) Wu, S. and Gebraeel, N. and Lawley, M. A. and Yih, Y. (2007). A Neural Network Integrated Decision Support System for Condition-Based Optimal Predictive Maintenance Policy, IEEE Transactions on Systems, Man, and Cybernetics - Part A: Systems and Humans, p. 226-236, Vol 37, Number 2

9) Wang, W. (2002). A model to predict the residual life of rolling element bearings given monitored condition information to date. IMA Journal of Management Mathematics, p. 3-16, Vol 13, Number 1

Paper sent to revision: 29.05.2012.

Paper ready for publication: 20.06.2012. 